\title{
A Target Alignment Algorithm for Through-the-Wall Radar Imagery Classification
}

\author{
Bijan G. Mobasseri \\ Electrical and Computer Engineering \\ Villanova University, PA 19085
}

\author{
Graeme E. Smith \\ Center for Advanced Communications \\ Villanova University, PA 19085
}

\author{
Imad Estephan \\ Electrical and Computer Engineering \\ Villanova University, PA 19085
}

\begin{abstract}
Sensing through the wall using radar is a valuable capability. There is considerable work in generating radar images of the interior of a room by beamforming of the radar backscatter generated in a Synthetic Aperture Radar (SAR) configuration. However, high level interpretation of the scene is a more difficult task. In previous work a minimum distance classifier was successfully used to recognize various targets placed in the scene. The approach suffered from the dependency of target features on target location. This work presents a solution to this problem by bringing the target intensity profiles into alignment with the training data prior to classification. The alignment is performed by moving the intensity profiles between locations using an Autoregressive Moving Average (ARMA) model. The classification results before and after alignment show a marked improvement.
\end{abstract}

\section{INTRODUCTION}

\section{A. Background To The Study}

Through the Wall Radar Imaging (TWRI) is at the forefront of modern radar technologies that may be applied in urban settings [1], [2]. The technology produces an image of the interior of a building, while the radar remains outside [3]-[7], and provides potentially vital intelligence to law enforcement officers, soldiers or rescuers in the event of a building collapse. Unfortunately, the radar images have little resemblance to optical images and comprehension of the imaged scene is difficult. It is desirable, therefore, to automate the classification of the TWRI content to assist in decision making.

When the TWRI target is moving, and is comprised of moving parts, Micro-Doppler Signature ( $\mu$-DS) classification can be employed. This technique has already been used to recognize personnel targets behind walls [8], [9]. However, there are many targets in the through-wall situation that do not move and so cannot be recognized by $\mu$-DS. Classification of these objects is necessary to provide the maximum intelligence about the scene beyond the wall. The intensity profile of a target in a TWRI changes depending on its position due to the antenna pattern and beamforming algorithm [3], [4]. This variation impedes classifier performance since target features are no longer fixed.

This paper investigates potential solutions to the problem of stationary target intensity signature variation in TWRI. The approach taken is to develop methods to align the signatures from the different positions within the scene to a single reference position. Once the target signature has been aligned, classification can be performed as if the targets are always (a) Position 1
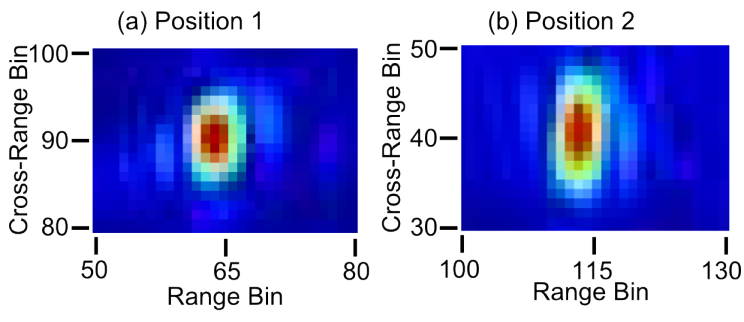

Fig. 1. Variation of TWRI with target position.

detected at the reference location. The principal alignment method employed is linear prediction [10], [11] and the classification technique used is a Minimum Mahalanobis Distance (MMD) classifier.

\section{B. Description Of The Problem}

Fig. 1 shows two 2D TWRI in which a metal sphere target is visible. The sphere has been imaged behind a wall, and the position of the sphere is different in each image. In part (a) the sphere is close to, and to the left of, the radar as well as being at a lower height than the sphere in part (b) that is further from, and to the right of, the radar. It was observed that the shape of sphere is different in the two TWRIs: it is smeared in cross-range in Fig. 1 part (b). This difference indicates a dependency of the target's intensity signature on location. The difference was further demonstrated using the MMD classifier reported on in [12]. The classifier was trained with target data from the nearer position (Fig. 1 part (a)). When presented with data from the near position target to recognize, the correct classification rate was $\approx 95 \%$. However, when presented with unlabelled data from the far position (Fig. 1 part (b)) the correct classification rate fell to $\approx 11 \%$. The drop in performance was attributed to the variation between the intensity profiles. This paper investigates how the variation could be compensated for prior to performing Automatic Target Recognition (ATR), and so improve classification performance.

Before turning to the proposed techniques, there is a further issue to consider: detection of stationary targets in TWRI. When a target is moving, it is relatively easy to separate it from station clutter through use of Moving Target Indication (MTI) [13], [14]. If the target is stationary, alternative methods have to be employed. Possible techniques include: an 


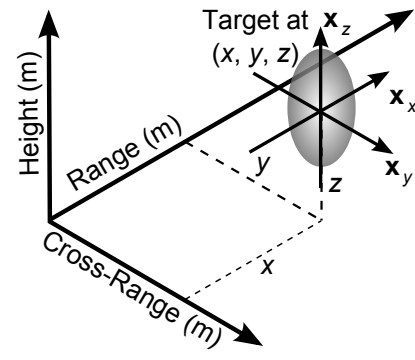

Fig. 2. Feature vectors in a 3D TWRI.

iterative version of the Neyaman-Pearson detector [15]; a minimum Mahanalobis distance classifier that can both detect and classify targets in one step [12]; and Iterative Conditional Modes (ICM) segmentation to divide the TWRI into target and non-target regions [16]. For this study emphasis was placed on the classification of targets that are assumed detected. To ensure a false alarm rate of zero, and allow testing of the proposed methods with independence from the detection strategy, detection of targets was performed manually using prior knowledge of the target locations.

\section{Proposed Method}

\section{A. Linear Prediction}

TWRI emphasizes the detection of targets inside buildings. Under these conditions the dimensions of the scene being imaged are in the order of tens of metres. Within such a small scene it was speculated that the variation of the targets' intensity signatures approximated to a linear process. Such processes can be modelled using a linear predictor [10], [11]. If the alignment process is regarded as a filter with unknown coefficients, the relationship between the input and output signals can be expressed as a difference equation:

$$
y(n)=-\sum_{k=1}^{p} a_{k} y(n-k)+G \sum_{l=0}^{q} b_{l} x(n-l), \quad b_{0}=1
$$

where $y(x)$ is the output signal, $x(n)$ is the input signal, $G$ is the gain and $a_{k}$ and $b_{l}$ are the filter coefficients. In (1) $p$ and $q$ are the number of feedback and feedforward coefficients respectively.

The linear predictor can be used to form a Moving Average (MA) or Autoregressive (AR) filter by only using some coefficients. An Autoregressive Moving Average (ARMA) filter may be produced by using all of the coefficients; it is this form of the linear predictor that was used in this study because it offers the maximum degrees of freedom for a linear filter. The coefficients were found using the Steiglitz-McBride method [17].

\section{B. Aligning TWRI Targets}

Fig. 2 shows a representation of a 3D TWRI containing a single target at a position $(x, y, z)$. Associated with the target class, $c_{i}$, is a set of feature vector, $\chi_{i, k}$, where:

$$
\chi_{i, k}=\left\{\mathbf{x}_{i, k}^{(1)}, \mathbf{x}_{i, k}^{(2)}, \ldots \mathbf{x}_{i, k}^{\left(n_{i, k}\right)},\right\}, k \in\{x, y, z\}
$$

and where $\mathbf{x}_{i, k}^{(n)}$ is the $n^{\text {th }}$ intensity profile along the $k^{\text {th }}$ dimension of class $c_{i}$. The targets of interest have an intensity volume that occupies more than one voxel in the TWRI; it is therefore expected that there will be several intensity profiles along each dimension for a particular target. The objective was to align the profiles so that they were as if the target location were at some reference position $\left(x_{\text {ref }}, y_{\text {ref }}, z_{\text {ref }}\right)$. It should be noted that the length of each intensity profile depends on the number of samples taken along its related dimension. As a result the individual dimensions must be processed separately and the classification results fused as a final step, this is the approach taken in [12], but without the alignment process.

Alignment is undertaken in two stages: first, a circular shift is used to rotate the peak of the intensity profile to the reference location; and second, the ARMA linear predictor is applied to compensate for the variation in intensity signature resulting from the target's original location. Performance of the alignment process may be assessed directly, without the need to undertake target classification. The normalized error, $\epsilon$, for the alignment process was described by,

$$
\epsilon=\frac{\left\|\mathbf{x}_{\text {ref }}-\mathbf{x}_{\text {align }}\right\|_{2}}{\left\|\mathbf{x}_{\text {ref }}\right\|_{2}}
$$

where $\mathbf{x}_{\text {ref }}$ is an intensity profile at the reference location, $\mathbf{x}_{\text {align }}$ is a aligned intensity profile from a detected target and $\|\cdot\|_{2}$ is the Euclidean norm.

\section{Alignment Results And Analysis}

\section{A. The TWRI Dataset}

The TWRIs used in this study were gathered in the Radar Imaging Laboratory of the Center For Advanced Communications, Villanova University. A $57 \times 57$ element array is synthesized using a single horn antenna. The horn is moved to each element position in the array in turn and, if the scene being imaged remains constant, the array can be synthesized in post processing [18]. The spacing between elements was $0.0215 \mathrm{~m}$ in both cross-range and height. The waveform was a $1 \mathrm{GHz}$ bandwidth, stepped frequency, continuous wave signal with a centre frequency of $1.5 \mathrm{GHz}$. This provided a range resolution of $0.15 \mathrm{~m}$. The system was used to gather a 3D intensity image of a scene behind $5 \mathrm{~cm}$ thick plywood wall, with the antenna at a stand off distance of $0.68 \mathrm{~m}$ from the wall. The image was formed using the beamforming method described in [3], and the voxel (volume pixel) edge length was $3.75 \mathrm{~cm}$ representing four times oversampling of the range resolution. To simplify the problem, the walls of the laboratory are lined with absorber to minimize multipath. Additionally, data for the empty scene was collected and subtracted from the target data. This further minimizes the effects of the laboratory walls and reduces the significance of the plywood wall. It is assumed that for an operational system there would be time during deployment to capture the empty scene prior target detection.

A set of three images was measured for two fundamental targets: a metal sphere of diameter $0.36 \mathrm{~m}$ and a metal dihedral with $0.39 \times 0.28 \mathrm{~m}$ faces. In the images the target is centred in 


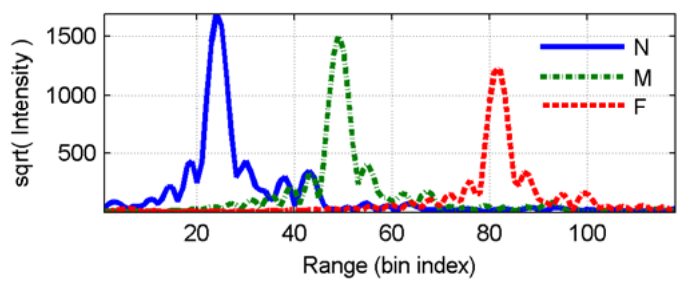

Fig. 3. Range intensity profile of the dihedral at the three different positions. $\mathrm{N}=$ Near, $\mathrm{M}=$ Middle $\& \mathrm{~F}=$ Far.

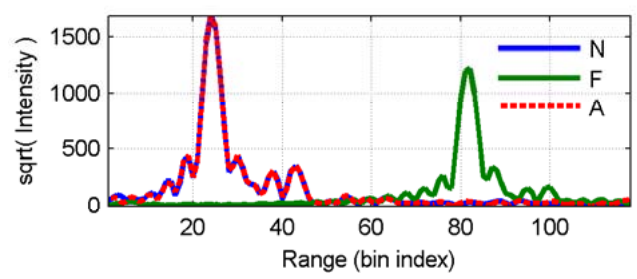

Fig. 4. The target alignment process. $\mathrm{N}=\mathrm{Near}, \mathrm{F}=$ Far $\& \mathrm{~A}=$ Aligned.

height ( $z$-axis) and cross-range ( $y$-axis) but is moved between three range positions. The near position is $0.6 \mathrm{~m}$ from the wall; the middle, $1.51 \mathrm{~m}$; and the far $2.73 \mathrm{~m}$. In this study, the near position was considered the reference and the investigation focused on aligning the middle and far targets to this position.

\section{B. Aligning The Signatures}

For each of the targets intensity profiles in the range, $x$, cross-range, $y$, and height $z$ dimensions were extracted. All of the profiles passed within the $-9 \mathrm{~dB}$ isocontour of the TWRI, which had been normalized to the peak target intensity. The profiles in each dimension were then averaged to provide a mean profile, from which the linear prediction filter coefficients could be derived. Fig. 3 shows the mean intensity profiles for the dihedral target at the three different ranges. Following the circular shifting of the middle and far profiles to align their peaks with the near profile, the Steiglitz and McBride algorithm [17] was used to find the coefficients for the ARMA filter. The optimum number of poles and zeros for the filter was found by performing an exhaustive search and looking for the combination that minimized the error, $\epsilon$. Different size filters were used for each target, position and dimension profile. The results for aligning the range dimension of the far dihedral target is shown in Fig. 4. In the figure: the blue line is the dihedral in the near position; the green line, the far position; and the red, dashed line the far signature after alignment to the near position. The aligned far signature is almost identical to the near signature with an error of $\epsilon=0.1$. Close observation of the aligned curve shows that it is nearly perfect through the mainlobe of the intensity profile, with the error emerging in the tails.

\section{Alignment With Swapped Coefficients}

To test the robustness of the filter coefficients two tests were conducted in which inputs were aligned using swapped coefficients. The first swap considered was based on the target
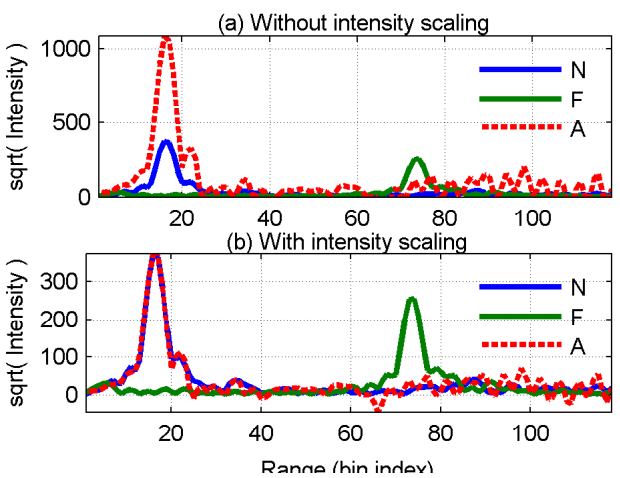

Fig. 5. The target alignment process for coefficients from different ranges. $\mathrm{N}=$ Near, $\mathrm{F}=$ Far $\& \mathrm{~A}=$ Aligned.

location: an input was filtered with the coefficients for the correct class of target, but from a different position e.g. filter a target at the middle position with the coefficients from the far position. This test investigated the robustness of the coefficients to the variation an intensity profile might exhibit based on the target location in the TWRI. The second test aligned a target using the coefficients designed for another class e.g. the dihedral target was aligned with the sphere's coefficients. When a target is first detected in a TWRI its class will be unknown and the input regarded as unlabelled. Understanding how to process unlabelled inputs is important for an operational system and the latter test results helped to define the processing strategy outlined in section III-D.

Fig. 5 shows the results for aligning the sphere target's range profile at the middle position with the coefficients of the far position. The colouring and labelling of the plots is the same as Fig. 4. In Fig. 5 part (a) it was observed that the amplitude of the aligned profile is much larger than the reference position. The error between the aligned and reference profiles was $\epsilon=$ 2.0. When a target is further from the radar, the power of the backscatter signal is less, according to the $R^{-4}$ law. The ARMA filter compensates for this, and since a nearer target is being aligned with a far target's coefficients there is too much intensity scaling. In Fig. 5 part (b) the intensity has been rescaled and it can be seen that the quality of the fit is much improved. $\epsilon=0.2$ and it is was noted that, again, the majority of the error was in the tails of the profile, not the main target.

Fig. 6 shows the results for aligning the dihedral target's range profile, at the far position, with the sphere's coefficients. In part (a) of the figure, where no scaling was used, the magnitude of the aligned profile is too large, resulting in an error of $\epsilon=2.0$; in part (b), when scaling is used, the aligned profile is too small, resulting in an error of $\epsilon=0.6$. The scaling problems resulted from the dihedral having a larger Radar Cross Section (RCS) than the sphere. The filter coefficients for the dihedral at the far position resulted in a gain, $G$ from (1), of 1.4, but for the sphere it was 3.8. When no scaling was used $G=3.8$ was too much causing the aligned signature to be to be too large. However, when scaling is used, the 


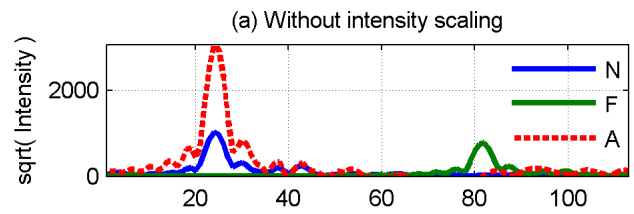

(b) With intensity scaling

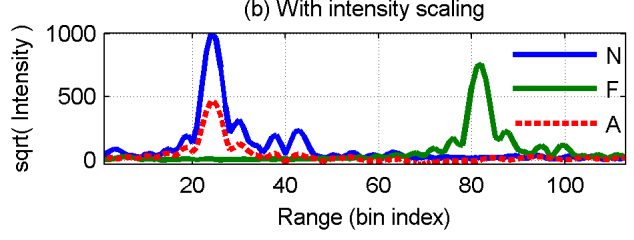

Fig. 6. The target alignment process for coefficients from different targets. $\mathrm{N}=$ Near, $\mathrm{F}=$ Far \& $\mathrm{A}=$ Aligned.

dihedral is scaled by the peak intensity of the sphere at the far position, since this intensity corresponded to the coefficients being used. The resulting scaled input profile had a much lower peak intensity than the original dihedral at this position and $G=3.8$ was not enough to properly align it with the dihedral at the near position.

\section{Effect Of Alignment On Classification}

The ultimate aim of the alignment process is to provide consistent feature vectors for classification. Above, a combination of circular shifting, combined with bespoke linear filter coefficients, provided very accurate correction of the distortions that arise in intensity profiles extracted from TWRIs due to the antenna pattern. Attention now turns to how best these results can support radar ATR. Two issues are considered here: first, how to select the appropriate coefficients for a given target location; and second how to process the the unlabelled input when it is not know which set of coefficients to apply.

While the results of section III-C indicated the linear filter approach to be robust to changes in target location, limitations of the test dataset must be considered. In the available data the targets move along the antenna boresight only. This results in a range profile that is somewhat invariant to position. It exhibits magnitude scaling with range, and shape changes only occur when the magnitude of features of the profile fall below the noise floor. Conversely, the cross-range and height profiles both exhibit dilation with increasing range, since the resolution in these dimensions depends on target range. If the target locations that included variation in cross-range and height had been used similar effects would have been observed in the range dimension as the system resolution cell orientation became different to that of the voxel grid due to steering the antenna pattern. To ensure optimum performance it is anticipated that a working system would need to have several sets of filter coefficients for different locations. During target detection an accurate measure of target position is made; this can then be used to select the appropriate coefficients. Even if many thousand sets of coefficients were required, since they are developed off line, it would not effect speed of operation.

The presented results also provide a method for determining
TABLE I

CORRECT CLASSIFICATION RATE WITHOUT ALIGNMENT.

\begin{tabular}{r|cc|cc|cc}
\hline \hline Profile: & \multicolumn{2}{|c|}{ Range } & \multicolumn{2}{c|}{ Cross-Range } & \multicolumn{2}{c}{ Height } \\
Declared as: & Sph & Dih & Sph & Dih & Sph & Dih \\
\hline Near Sphere & 100 & 0 & 100 & 0 & 100 & 0 \\
Mid Sphere & 0 & 100 & 100 & 0 & 100 & 0 \\
Far Sphere & 0 & 100 & 100 & 0 & 98 & 2 \\
Near Dihedral & 0 & 100 & 0 & 100 & 0 & 100 \\
G. & 0 & 100 & $\mathbf{7 0}$ & $\mathbf{3 0}$ & 0 & 100 \\
Mid Dihedral & 0 & 98 & 0 & 100 \\
Far Dihedral & 0 & 100 & 2 & 98 \\
\hline \hline
\end{tabular}

which filter coefficients to use for an unlabelled input. It was observed that the normalized error after alignment was minimized when the filter coefficients that match the input's class were used. Since even point targets occupy several voxels in the TWRI image, multiple intensity profiles can be extracted per detection, see section II-B. To accurately align an unlabelled target at an arbitrary position, all of the intensity profiles should be processed with all sets of coefficients and the resulting aligned profiles passed to the classifier. The set of coefficients that result highest declaration for a single class would then be regarded as the correct ones to use. This repetition of the classification process using different coefficients to obtain best performance, and so select coefficients, is analogous to the way data is tested under each hypothesis, to see which is most likely, in a maximum a posteriori approach such as a naïve Bayesian classifier.

\section{Classification Results And Analysis}

\section{A. Description Of Tests}

Testing was conducted using the MMD classifier that was proposed in [12]. First, Principal Component Analysis (PCA) was used to reduce the dimensionality of the intensity profile. The PCA processing kept $99.5 \%$ of the total variance which resulted in three components being kept for the range and height profiles and four for the cross-range. This was a significant reduction, since originally these profiles contained 113, 47 and 129 elements respectively. The covariance matrix of the data in its new basis was then computed for use in the MMD classifier. The near position was used as the reference position for the alignment process and to provide the training data. Of the available profiles, $40 \%$ were used to train the classifier and the remaining $60 \%$ held back for testing. From the other positions all the profiles were used for testing. The coefficients used for alignment were as calculated above.

\section{B. No Alignment Tests}

As a baseline, the performance of the classifier without using alignment is shown in Table I. The table details the rates, expressed as percentages, that each input was declared as a particular class for the different intensity profiles. For the range profile it was observed that, except for the case of the near sphere that was used to train the classifier, the declaration is always for dihedral. In the cross-range case only the middle dihedral, results shown in bold, was incorrectly classified with any significance while for the height profile the performance 
TABLE II

CORRECT CLASSIFICATION RATE WITH MATCHED ALIGNMENT.

\begin{tabular}{c|cc|cc|cc}
\hline \hline Profile: & \multicolumn{2}{|c|}{ Range } & \multicolumn{2}{c|}{ Cross-Range } & \multicolumn{2}{c}{ Height } \\
Declared as: & Sph & Dih & Sph & Dih & Sph & Dih \\
\hline Near Sphere & 100 & 0 & 100 & 0 & 100 & 0 \\
Mid Sphere & 100 & 0 & 100 & 0 & 100 & 0 \\
Far Sphere & 100 & 0 & 100 & 0 & $\mathbf{8 1}$ & $\mathbf{1 9}$ \\
$\Xi$ Near Dihedral & 0 & 100 & 0 & 100 & 0 & 100 \\
Mid Dihedral & 0 & 100 & 4 & 96 & 0 & 100 \\
Far Dihedral & 0 & 100 & 9 & 91 & 0 & 100 \\
\hline \hline
\end{tabular}

TABLE III

CORRECT CLASSIFICATION RATE WITH SWAPPED POSITION ALIGNMENT.

\begin{tabular}{r|cc|cc|cc}
\hline \hline Profile: & \multicolumn{2}{|c|}{ Range } & \multicolumn{2}{c|}{ Cross-Range } & \multicolumn{2}{c}{ Height } \\
Declared as: & Sph & Dih & Sph & Dih & Sph & Dih \\
\hline Near Sphere & 100 & 0 & 100 & 0 & 100 & 0 \\
Mid Sphere & 100 & 0 & 100 & 0 & 100 & 0 \\
Far Sphere & 91 & 9 & 100 & 0 & $\mathbf{2 8}$ & $\mathbf{7 2}$ \\
Near Dihedral & 0 & 100 & 0 & 100 & 0 & 100 \\
Mid Dihedral & 0 & 100 & $\mathbf{3 5}$ & $\mathbf{6 5}$ & 0 & 100 \\
Far Dihedral & 0 & 100 & 0 & 100 & 0 & 100 \\
\hline
\end{tabular}

was almost perfect. In addition, classification was performed using only circular shifting to align the peaks and circular shifting followed by signature normalization of the profile so that all peaks were one. These methods made no significant changes to the results shown in Table I.

\section{Matched Alignment Tests}

The optimum situation would be the case when the alignment is matched to the input that must be classified i.e. the coefficients for the correct range and correct class are applied. The results for this situation are shown in Table II and it can be seen that the classification performance was much improved. For the range profile the classifier always made the correct declaration while for the cross-range and height profiles only classification of the far sphere, using the height profile, was significantly different from perfect classification. In this case the classifier declared the right class at a rate of $81 \%$. The reduced performance was attributed to the far sphere having the smallest return power of the targets. The lower power return resulted in a low Signal to Noise Ratio (SNR) that would cause the target to appear different to the classifier.

\section{Mismatched Alignment Tests}

The classification testing was repeated using two mismatched alignment processes. In the first test, the filter coefficients for the incorrect target position, but correct target class, were used. For example, if the target was at the middle position then the coefficients for the far position were used and vice versa. In the second test the coefficients for the incorrect class, but correct target position, were used. For example, the sphere was always aligned with the dihedral's filter coefficients. Following the results of section III-C the input intensity profiles were scaled prior to alignment and classification.

The results for the swapped position test are shown in Table III. Observation of the table indicates a good correspon-
TABLE IV

CORRECT CLASSIFICATION RATE WITH SWAPPED CLASS ALIGNMENT.

\begin{tabular}{r|cc|cc|cc}
\hline \hline Profile: & \multicolumn{2}{|c|}{ Range } & \multicolumn{2}{c|}{ Cross-Range } & \multicolumn{2}{c}{ Height } \\
Declared as: & Sph & Dih & Sph & Dih & Sph & Dih \\
\hline Near Sphere & 100 & 0 & 100 & 0 & 100 & 0 \\
Mid Sphere & 100 & 0 & 100 & 0 & 100 & 0 \\
Far Sphere & $\mathbf{6 5}$ & $\mathbf{3 5}$ & $\mathbf{8 5}$ & $\mathbf{1 5}$ & $\mathbf{0}$ & $\mathbf{1 0 0}$ \\
Near Dihedral & 0 & 100 & 0 & 100 & 0 & 100 \\
G. Didhedral & 0 & 100 & $\mathbf{2 0}$ & $\mathbf{8 0}$ & 0 & 100 \\
Mid Dir Dihedral & 0 & 100 & $\mathbf{8 0}$ & $\mathbf{2 0}$ & 0 & 100 \\
\hline Far Dihel
\end{tabular}

TABLE V

DECLARATION OF UNLABELLED INPUT,

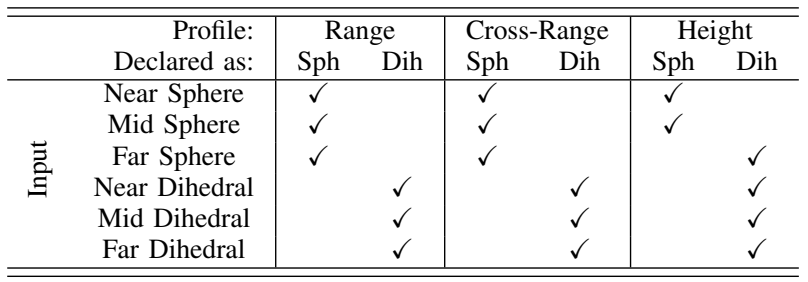

dence with the results of section III-C. There were only two targets with significant misclassification rates: the far sphere (as was the case when the alignment process was matched) and the middle dihedral. In section III-C it was observed that the normalized errors for intensity profiles aligned with filter coefficients from a different position were only marginally larger than when the optimum coefficients were used. The classification results showed a similar trend with only a slight drop in performance compared to the case where a matched alignment process was used. Furthermore, it was also observed that the majority of the error between the aligned and reference profile was in the noise regions and that the fit through the target region was particularly good. When this result was combined with the effects of PCA the resilience of the ATR process to swapping the coefficients could be completely understood. The PCA processing removes the noise regions from the intensity profiles. Since these regions are where the majority of the error in alignment was arising, improved performance was expected.

Table IV details the declaration rates for the case when the input is deliberately aligned with the coefficients for the other class. These results represent the worst performance observed with five instances where the classifier either tended to declare the wrong class, or where the correct classification rate was substantially different to $100 \%$. These results suggest that the coefficients are more closely linked to the targets' true classes than their positions, and support the proposed method for processing an input of unlabelled class from section III-D.

\section{E. Unlabelled Input Classification}

The final classification testing conducted investigated the ability of the target alignment approach to correctly identify a new detection in a TWRI for which there is no prior class label, the unlabelled input. The process by which such a detection might be identified was described in section III-D. The results are shown in Table V. This table is different to the 
others: it no longer lists classification rates. Instead it gives a single indication of which class was declared. This change is a result of the processing method that uses the multiple profiles extracted to decide which coefficients should be used, rather than to assess the classifier performance. Table $\mathrm{V}$ shows that in all but one case, the far position sphere being recognized based on height profile, the approach resulted in a declaration for the correct class. In the instance where the wrong class was declared, the results for the other two dimensions were for the correct class. If an overall declaration were made based on a maximum number of votes basis, then sphere would be declared based on the results for the three dimensions.

Underlying the simple boolean nature of Table V are several classification rates that have to be compared. For each set of coefficients applied, there is a resulting rate of declaration for each possible target class. In many of the situations tested here the result were unanimous with a rate of $100 \%$ for a particular class and $0 \%$ for the other when both sets of coefficients were applied. There were a few cases when the result was not so straightforward, however. When identifying the far dihedral using the cross-range profile and the sphere coefficients for alignment the declaration rate was $80 \%$ for the sphere. However, the dihedral coefficients gave a rate of $91 \%$ for the dihedral. (Note these rates don't add to $100 \%$ since they originate in different hypothesis.) This lead to uncertainty in deciding which was the correct set of coefficients to use since both $80 \%$ and $91 \%$ are high rates. In an operational system this dilemma could be overcome using two different approaches. First, the class finally declared could be required to have a declaration rate greater than the alternatives plus some offset, e.g. it must have a rate at $20 \%$ above the nearest alternative, as proposed in [19]. Second, the results from the other dimensions could be use to confirm that the right choice had been made.

\section{Summary And CONCLusions}

The significance of aligning targets in TWRI to a reference position prior to application of ATR has been demonstrated. The alignment was achieved by application of an ARMA linear predictor model to the target intensity profiles. A bespoke set of filter coefficients was required for each target class and dimension of the TWRI image. With appropriate scaling, the coefficients for the targets' range profiles were found to be robust to variations in the target location. The dependence of the alignment coefficients on the target class was overcome by repeated filtering with different sets of coefficients. The correct coefficients were regarded as those that gave the highest probability of declaration for a particular class.

Significant improvements in the correct classification rate were shown when using target alignment in conjunction with a MMD classifier. It was concluded that target alignment was a necessary step in the classification of targets within TWRI. The classification process also supported the alignment method. It was observed that the majority of the error between aligned and reference profiles was in noise regions and the PCA processing removed these regions effectively improving the alignment. From these observations it was concluded that the alignment and classification method should not be considered in isolation but as a symbiotic pairing.

The authors plan to continue researching this area to investigate how techniques from image registration can be applied to align the signatures and the effect of using more complex target structures.

\section{REFERENCES}

[1] D. D. Ferris and N. C. Currie, "Survey of current technologies for through-the-wall surveillance (tws)," in SPIE Conference on Sensors, C31 Information, and Training Technologies for Law Enforcement. Boston, MA, USA: SPIE, 1998, pp. 62-72.

[2] E. Baranoski, "Through wall imaging: Historical perspective and future directions," in Acoustics, Speech and Signal Processing, 2008. ICASSP 2008. IEEE International Conference on, 31 2008-April 4 2008, pp. 5173-5176.

[3] F. Ahmad and M. G. Amin, "Multi-location wideband synthetic aperture imaging for urban sensing applications," Journal of the Franklin Institute, vol. 345, no. 6, pp. 618-639, 2008, advances in Indoor Radar Imaging.

[4] F. Ahmad, Y. Zhang, and M. Amin, "Three-dimensional wideband beamforming for imaging through a single wall," Geoscience and Remote Sensing Letters, IEEE, vol. 5, no. 2, pp. 176-179, April 2008.

[5] F. Ahmad, M. Amin, and G. Mandapati, "Autofocusing of throughthe-wall radar imagery under unknown wall characteristics," Image Processing, IEEE Transactions on, vol. 16, no. 7, pp. 1785-1795, July 2007.

[6] F. Ahmad, M. Amin, and S. Kassam, "Synthetic aperture beamformer for imaging through a dielectric wall," Aerospace and Electronic Systems, IEEE Transactions on, vol. 41, no. 1, pp. 271-283, Jan. 2005.

[7] L.-P. Song, C. Yu, and Q. H. Liu, "Through-wall imaging (twi) by radar: 2-d tomographic results and analyses," Geoscience and Remote Sensing, IEEE Transactions on, vol. 43, no. 12, pp. 2793-2798, Dec. 2005.

[8] L. Du, J. Li, P. Stoica, H. Ling, and S. Ram, "Doppler spectrogram analysis of human gait via iterative adaptive approach," Electronics Letters, vol. 45, no. 3, pp. $186-188,292009$.

[9] S. Ram and H. Ling, "Through-wall tracking of human movers using joint doppler and array processing," Geoscience and Remote Sensing Letters, IEEE, vol. 5, no. 3, pp. 537-541, July 2008.

[10] J. Makhoul, "Correction to "linear prediction: A tutorial review"," Proceedings of the IEEE, vol. 64, no. 2, pp. 285-285, Feb. 1976.

[11] _ _Linear prediction: A tutorial review," Proceedings of the IEEE, vol. 63, no. 4, pp. 561-580, April 1975.

[12] Z. Rosenbaum and B. G. Mobasseri, "Interpretation of through-the-wall radar imagery by probabilistic volume model building," in SPIE Defense, Security And Sensing, Orlando, Florida, March 2008.

[13] N. Maaref, P. Millot, C. Pichot, and O. Picon, "FMCW ultra-wideband radar for through-the-wall detection of human beings," in Proceedings Of The 2009 International Radar Conference, Bordeaux, France, October 2009.

[14] M. I. Skolnik, Ed., Radar Handbook, 3rd ed. McGraw-Hill, 2008, ch. 2, pp. 2.1-2.102.

[15] C. Debes, M. Amin, and A. Zoubir, "Target detection in single- and multiple-view through-the-wall radar imaging," Geoscience and Remote Sensing, IEEE Transactions on, vol. 47, no. 5, pp. 1349-1361, May 2009.

[16] C. Debes, J. Hahn, A. M. Zoubir, and M. G. Amin, "Feature extraction in through-the-wall radar imaging," in IEEE International Conference on Acoustics, Speech and Signal Processing (ICASSP), Under Review.

[17] K. Steiglitz and L. McBride, "A technique for the identification of linear systems," Automatic Control, IEEE Transactions on, vol. 10, no. 4, pp. 461-464, Oct 1965.

[18] F. Ahmad, G. Frazer, S. Kassam, and M. Amin, "Design and implementation of near-field, wideband synthetic aperture beamformers," Aerospace and Electronic Systems, IEEE Transactions on, vol. 40, no. 1, pp. 206-220, Jan 2004

[19] G. E. Smith, M. Vespe, K. Woodbridge, and C. J. Baker, "Radar classification evaluation," in Proceedings Of The 2008 IEEE Radar Conference, Rome, May 2008, pp. 1585-1590. 